\title{
The COVID-19 pandemic, heart and cardiovascular diseases: What we have learned
}

\author{
Zuzanna Piątek ${ }^{1, D, F}$, Paweł Gać, ${ }^{2, E, F}$, Małgorzata Poręba ${ }^{3}, A, D, F$ \\ ${ }^{1}$ Vide Cor Meum Students' Scientific Circle at the Division of Pathophysiology, Wroclaw Medical University, Poland \\ ${ }^{2}$ Division and Department of Hygiene, Wroclaw Medical University, Poland \\ ${ }^{3}$ Division of Pathophysiology, Department of Physiology and Pathophysiology, Wroclaw Medical University, Poland \\ A - research concept and design; $\mathrm{B}$ - collection and/or assembly of data; $\mathrm{C}$ - data analysis and interpretation; \\ $D$ - writing the article; $\mathrm{E}$ - critical revision of the article; $\mathrm{F}$ - final approval of the article
}

Address for correspondence

Paweł Gá́

E-mail: pawelgac@interia.pl

\section{Funding sources}

None declared

Conflict of interest

None declared

Received on January 7, 2021

Reviewed on January 18, 202

Accepted on February 8, 2021

Published online on May 26, 2021

Cite as

Piątek Z, Gać P, Poręba M. The COVID-19 pandemic, heart and cardiovascular diseases: What we have learned. Dent Med Probl. 2021;58(2):219-227. doi:10.17219/dmp/133153

DOI

$10.17219 / \mathrm{dmp} / 133153$

Copyright

( 2021 by Wroclaw Medical University

This is an article distributed under the terms of the

Creative Commons Attribution 3.0 Unported License (CC BY 3.0)

(https://creativecommons.org/licenses/by/3.0/)

\begin{abstract}
In 2020, the coronavirus disease 2019 (COVID-19) pandemic overwhelmed the world, temporarily paralyzing healthcare and economic systems. Until now, we have learned a lot about the symptoms, pathophysiology, and complications of the disease as well as about the laboratory findings concerning the disease, and we are rapidly acquiring new data on the influence of COVID-19 on other aspects of human health beside its effects on the respiratory system. Patients with co-existing cardiovascular diseases (CVD) are more frequently hospitalized, more likely to be treated in an intensive care unit (ICU) and have poorer prognoses.

In this article, we discuss the impact of severe acute respiratory syndrome coronavirus 2 (SARS-CoV-2) on CVD, starting from the mechanisms connected with the renin-angiotensin-aldosterone system (RAAS) and the angiotensin-converting enzyme 2 (ACE2) receptors, and then describing the main pathologies in the heart and vessels detected in patients with COVID-19. Additionally, we comment on the problem of acute coronary syndrome (ACS), which may be triggered by viral infection. Finally, we discuss how, in some countries, the pandemic has changed treatment patterns, lowering the rate of invasive diagnostics and even falsely reducing the prevalence of CVD as a result of patients' fear of being admitted to hospital.
\end{abstract}

Keywords: cardiovascular diseases, COVID-19, SARS-CoV-2 


\section{Introduction}

Since December 2019, when the first case of viral pneumonia was reported in Wuhan, China, coronavirus disease 2019 (COVID-19) has become a global pandemic. As of December 17, 2020, there were 45,942,902 confirmed cases of COVID-19, including 1,192,644 deaths worldwide according to the World Health Organization (WHO). ${ }^{1}$ In Poland alone, as of December 17, 2020, there were 62,731 confirmed cases of COVID-19, including 5,631 deaths. ${ }^{2}$ COVID-19, which is caused by a novel RNA coronavirus known as severe acute respiratory syndrome coronavirus 2 (SARS-CoV-2), is primarily associated with severe acute respiratory syndrome (SARS), yet the respiratory system is not the only system affected. Studies have shown that many patients have comorbidities, such as hypertension, other cardiovascular diseases (CVD), diabetes, and chronic obstructive pulmonary disease (COPD), which contribute to a poorer prognosis. ${ }^{3}$

The relationship between CVD and COVID-19 is important for many reasons; at first glance, it is clear that COVID-19 patients with pre-existing CVD show a higher mortality rate than those without CVD. ${ }^{4}$ Patients with various cardiac disorders are also hospitalized and receive treatment in an intensive care unit (ICU) more often. Wang et al. reported that $58.3 \%$ of patients hospitalized due to COVID-19 had been previously diagnosed with hypertension and $25 \%$ had heart disease..$^{5}$ Additionally, the presence of CVD risk factors, such as obesity, increases mortality because of COVID-19, which is 3-5-fold higher in obese patients. ${ }^{6,7}$ On the other hand, some studies reported that $7.2-12 \%$ of COVID-19 patients suffered from newly detected acute cardiac injury with an increase in the troponin I concentration and $16.7 \%$ developed arrhythmias. ${ }^{5,8}$ Moreover, some authors suggest that COVID-19 patients may present symptoms of acute coronary syndrome (ACS), including individuals without pre-existing cardiac conditions. ${ }^{9}$ Furthermore, symptoms of pre-existing CVD, such as heart failure and coronary artery disease, may overlap with symptoms typical of COVID-19, as in the case of dyspnea, which is a key feature of heart failure. ${ }^{10}$

In patients with coronary artery disease, the inflammatory state may destabilize atherosclerotic plaques, which may worsen a patient's condition and prognosis. ${ }^{11,12}$ In some COVID-19 patients, the so-called 'cytokine storm' was detected, with higher levels of cytokines in blood, which could potentially be one of the pathophysiological factors that induce further cardiac complications. ${ }^{8,13}$

Some authors suggested that COVID-19 was associated with myocarditis, as the post-mortem biopsies of the individuals infected with SARS-CoV-2 revealed scarce amounts of mononuclear inflammatory infiltrates in the interstitial space of the myocardium. ${ }^{14}$ However, data is still limited, as the diagnosis of myocarditis in patients is mainly based on cardiovascular magnetic resonance imaging (CMR) and echocardiography. ${ }^{15,16}$ Myopericarditis in COVID-19 patients sometimes leads to cardiac tamponade in the course of ACS and even in cases of unobstructed coronary arteries. ${ }^{17,18}$ This problem is still under investigation, and different tests, not only employing the histological analysis, but also identifying changes in the CMR images of the heart, should be recommended, as endomyocardial biopsies are not available in most COVID-19 cases.

The angiotensin-converting enzyme 2 (ACE2) receptor, which is widely expressed in the heart, has been found to have a strong binding affinity for the SARS-CoV-2 spike protein. ${ }^{19,20}$ This has led to the hypothesis that ACE2 may mediate cardiac injury in COVID-19 patients; however, recent studies on the hospitalized patients with hypertension showed a lower risk of all-cause mortality in the group of ACE inhibitor (ACEI)/angiotensin receptor blocker (ARB) (drugs that act through the abovementioned receptors) users as compared to non-users. ${ }^{21}$

Research studies are being conducted rapidly and new data is published nearly every week, yet key questions remain unanswered, including questions regarding the relationship between tissue and biochemical changes and the clinical condition of patients. We performed a systematic analysis of research articles published in medical journals between February 2020 and December 2020, searching the PubMed, Google Scholar and ScienceDirect databases with the use of keywords such as "COVID-19" OR "SARS-CoV-2" AND "cardiovascular disease", "ACE2 receptor", "heart", "obesity", "myocarditis", "arrhythmia”, "atrial fibrillation", "acute coronary syndrome", "thromboembolism", and "hypertension". Then, we studied the current available data about patients suffering from various cardiological disorders in the era of the lasting pandemic, with a particular focus on the potential impact of the virus on the heart. The aim of this review was to provide an up-to-date synopsis of the influence of COVID-19 on the heart and its interactions with CVD by analyzing the SARS-CoV-2 pathogenesis, clinical complications and prognosis in patients with and without co-existing CVD.

\section{General symptoms and pathologies of SARS-CoV-2}

The incubation period of the novel coronavirus is estimated to be between 3-7 days and up to 2 weeks in some cases. Like other respiratory viruses, SARS-CoV-2 spreads primarily through respiratory droplet transmission as well as through hands and surfaces. The initial infection may be manifested through fever, dry cough, fatigue, dyspnea, myalgia, sore throat, headache, and conjunctivitis; hyposmia and dysgeusia are sometimes reported, and diarrhea, nausea and vomiting also occur in rare instances. ${ }^{10,22,23}$ Most patients demonstrate mild 
flu-like symptoms; some develop pneumonia and, in around $5 \%$ of cases, severe acute respiratory distress syndrome (ARDS) and multi-organ dysfunction. However, the disease may also be asymptomatic. ${ }^{22,24}$ Indeed, it has been found that in about $80-90 \%$ of infected people, symptoms are mild or not present at all. ${ }^{22}$ Additionally, it has been shown that the majority of individuals with symptoms and more severe clinical patterns have 1 or more co-existing medical conditions, including hypertension, other CVD, diabetes, and chronic lung diseases. ${ }^{23,24}$ The mortality rate for COVID-19 is usually between $3 \%$ and $5 \%$, and out of the hospitalized patients, $10-20 \%$ are admitted to ICU, $3-10 \%$ require intubation and $2-5 \%$ die, as determined by Guan et al. ${ }^{25}$

Mounting evidence suggests that COVID-19 simultaneously causes multiple changes in other organ systems. Abnormal liver test results and liver damage are highly associated with severe pneumonia. ${ }^{10,22,24,25}$ Jaundice has been noted as a long-term complication of the SARS-CoV-2 infection, and nausea, vomiting, diarrhea, and abdominal pain are also common in COVID-19 patients, yet these gastrointestinal symptoms are not correlated with the severity of the disease. The positive results of the real-time polymerase chain reaction (RT-PCR) tests for SARS-CoV-2 in patients' stools raised suspicion for fecaloral transmission. Moreover, even after obtaining a negative throat swab test result, the virus was still detectable in feces. ${ }^{26}$ This possible route of transmission remains to be examined. It is also known that the mortality rate is directly proportional to age, reaching almost $30 \%$ fatality in patients over 80 years old, although death can occur at any age, including children and newborns. ${ }^{3,22,24,25,27,28}$

In laboratory tests from COVID-19 patients, we find leukocytosis or leukopenia, often with lymphopenia, which is an effective indicator of the severity of the infection. The alanine aminotransferase, aspartate aminotransferase and $\mathrm{C}$-reactive protein (CRP) levels are increased as well. ${ }^{3,10,22,24,25,28}$ Furthermore, increased troponin levels were also reported in $7 \%$ of patients who died from fulminant myocarditis, and some authors suggested it was a strong prognostic indicator of mortality, similar to the CRP level. In addition, the D-dimer and ferritin levels

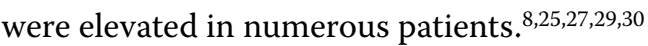

\section{Possible pathophysiological mechanisms of cardiovascular complications in COVID-19 patients}

The mechanisms through which SARS-CoV-2 affects the cardiovascular system are still under investigation. However, there is some data on its interactions with the renin-angiotensin-aldosterone system (RAAS). Angiotensin-converting enzyme 2 has been identified as crucial in facilitating the entry of SARS-CoV-2 into the host cell and it is highly expressed in alveolar epithelial type 2 (AT2) cells. The virus exhibits binding to the cell-associated and soluble ACE2 receptors, expressed in numerous organs, such as the heart, kidneys, intestine, lung, brain, and liver, ${ }^{31,32}$ which may explain why COVID-19 patients manifest a spectrum of symptoms, including gastrointestinal, cardiac and renal injury, along with neurological and respiratory symptoms. ${ }^{33}$ Through the respiratory tract, SARS-CoV-2 reaches AT2 cells, which are responsible for surfactant production. ${ }^{34}$ Once the virus enters the host cell, its mediators activate macrophages to release cytokines and chemokines, which increase vasodilation and vascular permeability. Excess plasma compresses alveoli and impairs surfactant production; thus, alveoli collapse and the gas exchange is not fully efficient. Angiotensin-converting enzyme 2, which is widely expressed throughout the body, normally shows protective effects against CVD by transforming angiotensin II into beneficial angiotensin 1-7. ${ }^{35,36}$ The binding of SARS-CoV-2 to the ACE2 receptor induces the endocytosis of the complex, and thereby downregulates enzyme expression so that it is unable to exert organ-protective effects, which ultimately results in the reduction of the beneficial anti-inflammatory properties and vasodilation associated with ACE2. . $^{31,37}$

Severe acute respiratory syndrome coronavirus 1 (SARS-CoV-1), the virus responsible for the SARS epidemic in 2002-2004, and SARS-CoV-2 both act via RAAS through ACE2, an enzyme that physiologically counters the activation of RAAS, but also functions as a receptor for both SARS viruses. These effects have been discussed primarily in the context that widely used ACEI and ARB drugs could potentially enhance the spread of SARS-CoV-2 in alveoli and myocardial cells. However, the RAAS blockade has been shown to limit exposure to the SARS-CoV-1 spike protein, which normally induces acute lung injury in experimental mouse models; furthermore, data suggests that ACEI and ARB therapy may be protective and result in lower levels of interleukin 6 (IL-6), and increased CD3 and CD8 $\mathrm{T}$ cell counts in blood. ${ }^{31,38-41}$ Vaduganathan et al. claim that the withdrawal of RAAS inhibitors may be harmful in certain high-risk patients with known or suspected COVID-19, but globally, it is widely accepted that we do not yet have a complete understanding of the pathophysiological mechanism of the SARS-CoV-2 infection and lack clear therapeutic implications; moreover, the data referring to humans is too scarce to support or refute these hypotheses and concerns. ${ }^{37}$

The activation of RAAS ultimately stimulates the secretion of transforming growth factor beta (TGF- $\beta$ ), which is involved in fibrosis, cardiomyocyte apoptosis and cardiac enlargement. ${ }^{42}$ In some cases, viral infection with SARS-CoV-2 leads to the so-called 'cytokine storm', and higher concentrations of IL-6, interleukin 8 (IL-8), interleukin 10 (IL-10), and tumor necrosis factor alpha (TNF- $\alpha$ ) were reported in deceased patients as compared 
to those who recovered. ${ }^{43,44}$ Usually, the immune system counterbalances abrupt cytokine release through the activation of the ACE2/Mas receptor (MasR) axis; however, in COVID-19, this mechanism is diminished due to ACE2 downregulation. ${ }^{45}$ As a result of the previously mentioned RAAS imbalance, SARS-CoV-2 leads to endothelial dysfunction, ${ }^{46}$ as the endothelium lines the lumen of blood vessels, and is constantly exposed to direct viral invasion and massive cytokine release. The resulting endothelial injury impairs nitric oxide synthesis, which enhances vasoconstriction and thrombosis, and generally promotes CVD and atherosclerosis. Further examinations should be continued, as our understanding of the pathological mechanisms concerning COVID-19 is still limited.

\section{Heart and cardiovascular diseases in times of pandemic}

The impact of COVID-19 on the cardiovascular system has been widely reported. As summarized by Dhakal et al., cardiac complications in COVID-19 include acute myocardial injury, arrhythmias, cardiogenic shock, and even sudden death. ${ }^{33}$ In addition, drug interactions with COVID-19 therapy may cause arrhythmias, cardiomyopathy and sudden death. ${ }^{33}$

Generally, patients with chronic diseases, such as CVD, are at higher risk of developing serious complications and dying from COVID-19, and the risk increases with age. Elderly patients with co-existing comorbidities are more likely to require admission to ICU and the cardiac manifestations of CVD may contribute to higher overall mortality. ${ }^{47,48}$ Cardiovascular pathologies are present in 8-25\% of COVID-19 patients, with a higher proportion of patients with a worse prognosis. ${ }^{49}$ In Chinese studies of 1,527 hospitalized COVID-19 patients, about $17 \%$ showed hypertension, $14.4 \%$ had cardio-cerebrovascular diseases and $8-10 \%$ had diabetes ${ }^{30}$; similarly, in other studies, $2 / 3$ of patients had diabetes, CVD or cancer. ${ }^{30}$

The link between smoking and COVID-19 is interesting. There is no doubt that tobacco smoke causes the inflammation of the airways and is a major risk factor for CVD, yet articles on the relationship between smoking and COVID-19 are limited and ambiguous. There is, for example, evidence that active smoking does not correlate with the severity of COVID-19; however, another systematic review points out that current or former smoking is associated with a poorer prognosis than in the case of non-smokers. ${ }^{50-52}$

COVID-19 patients with diabetes and obesity more commonly have respiratory dysfunction due to low muscle strength, and are more prone to infections; therefore, they often require mechanical ventilation. Diabetes and pre-diabetes are also important risk factors in COVID-19 patients, as the high glucose levels and various additional conditions observed in this group of patients, such as increased thrombosis, inflammation and reduced lung capacity, significantly increase the risk of lethal outcomes as compared to non-diabetic patients. Additionally, a study published in "The Lancet" points to a mutual interaction - patients with diabetes are at greater risk of severe infection, and COVID-19 exacerbates hyperglycemia and can even cause diabetes de novo.

\section{Myocardial injury and COVID-19}

Elevated cardiac biomarkers, and especially an elevated level of troponin I in the high-sensitivity cardiac troponin test, are the main diagnostic values that determine admission to ICU. Various studies had been analyzed and it was found that myocardial injury, defined as an elevation in the cardiac troponin concentration above the $99^{\text {th }}$ percentile of the upper reference limit, was observed in 7-17\% of the hospitalized COVID-19 patients..$^{23,33,51}$ Moreover, the incidence of myocardial injury increases with the severity of the SARS-CoV-2 infection, rising to $22.2 \%$ of patients needing ICU care and $59 \%$ of patients who die from the disease. Additionally, in ICU patients, the hypersensitive troponin I concentration was statistically significantly higher than in the non-ICU group. ${ }^{5,23}$ In studies of early cases of COVID-19 in China, Wang et al. showed that among 138 patients, the most common cardiac complications during hospitalization were arrhythmia (23 patients) and acute cardiac injury (10 patients). ${ }^{5}$

\section{Acute coronary syndrome}

In the context of ACS, viral infections (e.g., influenza) are considered the potential triggers of ST-elevation myocardial infarction (STEMI). It is believed that the same applies to the SARS-CoV-2 infection. Chest pain and electrocardiogram (ECG) changes typical of ACS are among the initial manifestations of COVID-19, and diagnoses range from STEMI to Takotsubo cardiomyopathy. ${ }^{23,33,53,54}$ In a retrospective study performed in Lombardy, Italy, 28 cases of SARS-CoV-2-positive patients with confirmed STEMI were investigated. ${ }^{55}$ In 24 of the cases, the first clinical symptom was ST-elevation, which was detected before patients received the results of their SARS-CoV-2 tests. The rest (4 patients) developed ST-elevation during hospitalization. ${ }^{55}$ Data acquired from New York hospitals presented 18 patients with COVID-19 and STEMI, and out of this group, 10 patients were diagnosed at the time of admission and 8 patients had myocardial infarction later during hospitalization. ${ }^{53}$ Additionally, 33\% of patients did not have significant changes within the coronary arteries and were included in the non-coronary myocardial injury group; the mean age was 63 years..$^{53}$ 
The pandemic has also changed the dynamics of cardiovascular procedures. During the first weeks of the contagion, reduced admission of STEMI patients was observed, and data also suggests increased mortality, which was related not only to COVID- $19.5^{55}$ The example of Madrid shows us that the newest situation lowered the number of percutaneous coronary interventions (PCIs) by half and increased the usage of fibrinolysis. ${ }^{56}$ In Saudi Arabia, the weekly and monthly hospitalization rates were significantly cut down as compared to previous years. ${ }^{57}$

With regard to the pathophysiological mechanism, it is possible that myocardial ischemia and infarction could be secondary to the plaque rupture triggered by the virusinduced stress response or the exaggerated tendency to coagulation due to general infection, which is similar to the situation observed in the complications of influenza. ${ }^{30}$

\section{Myocarditis}

Viral infections are a common cause of myocarditis; the possible mechanism includes intensified cytokine release by pathological $\mathrm{T}$ cells and monocytes. Studies have shown that in several cases, fulminant myocarditis was a result of the complications of the SARS-CoV-2 infection, but none of them revealed viral particles in myocytes. Clinical manifestations in those cases included chest pain, dyspnea and fatigue. ${ }^{58}$

We have gained some information from the autopsies and endomyocardial biopsies of the patients infected with SARS-CoV-2. In a study on severely ill COVID-19 patients, cardiomyocyte hypertrophy, degeneration and necrosis were noted with mild interstitial hyperemia as well as edema with the infiltration of lymphocytes, monocytes and neutrophils. ${ }^{59}$ In another study, low-grade interstitial and endocardial inflammation as well as viral particles were observed in the cytopathic, structurally damaged interstitial cells, which demonstrated the loss of the cytoplasmic membrane integrity, but, although the inflammatory cells and myocytes are closely adjacent, no viral particles were observed in myocytes. ${ }^{60}$ The authors commented that cardiac myocytes showed non-specific damage, mainly characterized by focal myofibrillar lysis, but no cytopathic endothelial or small intramural vessel inflammation or thrombosis were noted; additionally, the authors claimed that more research studies were needed. ${ }^{60}$ In yet another report, scattered individual myocyte necrosis was observed, with lymphocytes adjacent to, but not surrounding the necrotic myocytes, and degenerating myocytes were also present, possibly representing an early manifestation of viral myocarditis. ${ }^{61}$ Tavazzi et al. suggested that myocardial injury in patients with the SARS-CoV-2 infection could be multifactorial, which was generally understood as numerous changes, such as atherosclerotic plaque rupture, coronary vasospasm, hypoxic injury to the vasculature, direct endothelial damage, or the formation of microthrombi. ${ }^{60}$
As previously mentioned, acute virus-negative lymphocytic myocarditis has been associated with the SARSCoV-2 respiratory infection, and reports show an improvement in cardiac biomarkers after treatment with lopinavir/ritonavir and hydroxycholoroquine. ${ }^{58,62}$ Aberrations in cardiac biomarkers and ECG changes occur in myocarditis, but they are not entirely specific to this cardiomyopathy. Laboratory findings, such as elevated lactate, abnormal values of erythrocyte sedimentation rate and inflammatory markers (e.g., CRP), could be helpful at early stages of myocarditis management.

It is still not clear whether SARS-CoV-2 exerts cardiotropism, as some authors suggest; at the same time others deny it. The final recognition is typically based on an endomyocardial biopsy, which is the gold standard for identifying myocarditis. It is possible that future studies that would include MRI of the heart in patients after COVID-19 could help us to better understand the complex pathologies in this matter.

\section{Arrhythmias}

It is known that the elevated cytokine levels and the systemic inflammatory response which accompany myocardial injury predispose patients to atrial and ventricular arrhythmias. Cardiac manifestations in some viral infections include atrial fibrillation (AF), supraventricular tachycardia (SVT) and ventricular tachycardia (VT). There are reports of rhythm disturbances in COVID-19 patients, ranging from sinus tachycardia to ventricular arrhythmias.

The potential mechanisms of AF in COVID-19 patients include the previously mentioned decrease in ACE2 receptor expression, cytokine storm, endothelial dysfunction, $\mathrm{T}$ cell exhaustion, and various kinds of electrolyte imbalance, like hypokalemia, which are common in COVID-19 patients and may be the trigger of AF induction. ${ }^{63}$

In a retrospective cohort study of 1,284 patients, 170 patients had elevated cardiac troponin I, and of that group, 44 patients had atrial tachycardia (AT) or AF (35 patients), ventricular arrhythmias (2 patients), or both atrial and ventricular arrhythmias (7 patients). ${ }^{64}$ Only 4 out of 25 patients who developed AT/AF during infection had a prior history of AF, which suggests that it could be a result of the SARS-CoV-2 infection. Ventricular rhythm disturbances proved to be fatal in 6 cases. ${ }^{64}$ Atrial fibrillation was also described in other studies; in a multi-center study of remdesivir therapy, among a group of 53 patients with a median age of 67 years whose data were analyzed, 34 patients were invasively ventilated and 2 patients had AF (6\%). ${ }^{65}$

In another study of 138 hospitalized COVID-19 patients, arrhythmia was diagnosed in $16.7 \%$ of patients, with a higher incidence in ICU patients as compared to non-ICU patients $(44.4 \%$ vs $6.9 \% ; p<0.001) .{ }^{5}$ Transient 
complete heart block was also reported in patients with critical COVID-19, who later died due to severe respiratory failure. ${ }^{66}$

At this moment, we cannot describe arrhythmias as primary COVID-19 complications per se, as it is difficult to discern if they are a result of the SARS-CoV-2 infection alone, general viral infection, or a side effect of treatment drugs. Drug interactions should also be considered and followed, as prolonged QTc, VT, and even lethal arrhythmias, like torsade de pointes, have been observed in patients taking hydroxychloroquine and azithromycin. Such interactions will certainly be described with time, as more and more studies on the complications and treatment of COVID-19 are conducted.

\section{Venous thromboembolism}

The laboratory data of COVID-19 patients confirm coagulation abnormalities. Reports include reduced platelet count, elevated D-dimer and fibrinogen levels, and prolonged prothrombin time, which are associated with a poorer prognosis. ${ }^{25}$ The exaggerated production of procoagulants, severe hypoxia and inflammation lead to a hypercoagulable state, which promotes thrombus formation. In the previous studies of infection with other coronaviruses, pulmonary artery thrombosis was rarely reported, whereas with SARS-CoV-2, it is quite different. ${ }^{67}$ In a recent study, pulmonary embolism (PE) was verified with pulmonary computed tomography (CT) angiograms in 32 of 106 patients. ${ }^{68}$ Additionally, in a prospective cohort study, deep-vein thrombi were revealed in 7 of 12 cases in whom deep-vein thrombosis (DVT) was not suspected prior to death. ${ }^{69}$ In 4 cases, thrombi from lower extremities induced PE, which was believed to be the direct cause of death. ${ }^{69}$ Further studies showed changes in small pulmonary vessels as well, including microthrombi. ${ }^{70,71}$

An observation of 184 ICU patients with a mean age of 64 years demonstrated a strong correlation between COVID-19 and DVT and PE events. ${ }^{72}$ Klok et al. found a $31 \%$ incidence of thrombotic complications in ICU patients with COVID-19, which is remarkably high, even though in all cases, standard doses of thromboprophylaxis were used, based mainly on nadroparin given subcutaneously. ${ }^{72}$ The observed thrombotic complications included primarily PE (25 cases), with additional reports of proximal DVT of the leg (1 case), catheter-related upper extremity thrombosis (2 cases) and ischemic stroke (3 cases). The authors recommend the regular control of the D-dimer and fibrinogen levels, along with pharmacological thrombosis prophylaxis. ${ }^{72}$

In an article concerning coagulopathy, Iba et al. underlined that the clinical presentation of COVID-19associated coagulopathy was primarily organ dysfunction, whereas hemorrhagic events were less frequent. ${ }^{73}$ Moreover, they compared bacterial sepsis-associated coagulopathy/disseminated intravascular coagulation (DIC) with COVID-19 consequences and found that in COVID-19 cases, pathologies like the prolongation of prothrombin time, activated partial thromboplastin time and a decrease in antithrombin activity were less frequent, and thrombocytopenia was relatively uncommon. However, thrombocytopenia was described in some cases, which the authors suggested might be caused, at least in part, by treatment with heparin. ${ }^{74}$

The mechanism of coagulopathy in COVID-19 needs to be further examined, but for now we can say that it follows Virchow's triad, which consist of endothelial dysfunction, hypercoagulability and the stasis of the blood flow. Together, these effects contribute to a greater thromboembolic risk and lethal complications (e.g., DIC).

\section{Treatment considerations}

Generally, recommendations for the treatment of cardiovascular complications in patients with COVID-19 are not different from those from the pre-COVID-19 era. In the cited studies, mechanical ventilation was used when needed in severely ill patients with low blood saturation, vasopressor catecholamines were introduced to elevate decreased blood pressure in case of shock, and in many other clinical conditions, the use of drugs and specific procedures was similar. ${ }^{47}$ However, some minor changes have been observed in ACS treatment in relation to the problem of handling the infected patient. The pandemic has created new challenges with regard to treating myocardial infarction, but the main management should not be changed. In case of STEMI, both SARS-CoV-2-negative and SARS-CoV-2-positive patients should be treated similarly, as any delay in obtaining test results can be harmful. ${ }^{75}$ It has been recommended that, if a patient is referred to a non-COVID-19-trained lab or a non-PCIcapable hospital, and a transfer to a COVID-19-trained/ PCI-capable facility can happen within $120 \mathrm{~min}$, it should be initiated, yet if it cannot happen within $120 \mathrm{~min}$, thrombolysis should be performed instead, with concomitant medical management with aspirin, clopidogrel, heparin, or enoxaparin, which generally meets the guidelines. ${ }^{75}$ However, it should be noted that some patients may have contraindications to thrombolysis. Additionally, Zheng et al. suggests that drug-related heart damage during COVID-19 treatment is a concern, and that the use of antiviral drugs in particular should be monitored. ${ }^{48}$ Such drugs are known to induce various cardiovascular disorders; cardiac insufficiency and arrhythmias, including sick sinus syndrome, have been observed after interferon and ribavirin. Thus, to avoid cardiac toxicity, antiviral dosing and side effects should be under control, particularly in patients with pre-existing CVD. ${ }^{48,76}$

As the pandemic continues, we will gain more knowledge and it may, with time, change some treatment regimens, 
Table 1. Main cardiovascular complications in patients with COVID-19

\begin{tabular}{|c|c|c|c|}
\hline References & $\begin{array}{l}\text { Patients } \\
\qquad(N)\end{array}$ & $\begin{array}{l}\text { Severity of the disease } \\
\qquad(n)\end{array}$ & $\begin{array}{l}\text { Cardiac event } \\
\qquad(n)\end{array}$ \\
\hline Wang et al. ${ }^{5}$ & 138 & $\begin{array}{c}\text { ICU (36) } \\
\text { non-ICU (102) }\end{array}$ & $\begin{array}{c}\text { arrhythmia (16) } \\
\text { acute cardiac injury (8) } \\
\text { arrhythmia (7) } \\
\text { acute cardiac injury (2) }\end{array}$ \\
\hline Si et al. ${ }^{64}$ & 1,284 & severe COVID-19, hospitalized & arrhythmia (44) \\
\hline Bangalore et al. ${ }^{53}$ & 18 & hospitalized & $\begin{array}{c}\text { myocardial infarction (8) } \\
\text { non-coronary myocardial injury (10) }\end{array}$ \\
\hline Meyer et al. ${ }^{54}$ & 1 & hospitalized & Takotsubo syndrome (1) \\
\hline Sala et al. ${ }^{58}$ & 1 & hospitalized & myocarditis (1) \\
\hline Tavazzi et al. ${ }^{60}$ & 1 & hospitalized & cardiogenic shock (1) \\
\hline Klok et al. ${ }^{72}$ & 184 & severe COVID-19, hospitalized & thrombotic event (57) \\
\hline Léonard-Lorant et al. ${ }^{68}$ & 106 & COVID-19, hospitalized & PE (32) \\
\hline Wichmann et al. ${ }^{69}$ & 12 & COVID-19-positive deaths & deep-vein thrombi (7) \\
\hline
\end{tabular}

ICU - intensive care unit; COVID-19 - coronavirus disease; PE - pulmonary embolism.

which currently do not differ from the mainstream cardiovascular guidelines. One example is based on the finding that hypokalemia is a common metabolic abnormality in COVID-19 patients, which is probably secondary to the reduction of the albumin levels; thus, in this context, the antihypertensive medications known to increase the serum levels of potassium (including carvedilol and eplerenone) should be a first-line choice in COVID-19 patients with co-existing arterial hypertension. ${ }^{77}$

\section{Conclusions}

After nearly a year of the COVID-19 pandemic, we have gathered new knowledge on the symptoms, complications and pathophysiological mechanisms of this disease, and its impact on human health. We have learned not only how it affects the respiratory system, but also about other pathologies, including changes in blood and some alterations in the cardiovascular system (Table 1). Initial studies suggest an interaction with RAAS; however, we believe that new research is still needed.

\section{ORCID iDs}

Zuzanna Piątek (10) https://orcid.org/0000-0002-0091-2806

Paweł Gać (10) https://orcid.org/0000-0001-8366-0239

Małgorzata Poręba (1) https://orcid.org/0000-0002-4868-3088

\section{References}

1. World Health Organization. WHO Coronavirus (COVID-19) Dashboard. https://covid19.who.int/table. Accessed December 17, 2020.

2. Polish Council of Ministers. Report on infections with SARS-CoV-2 [in Polish]. https://www.gov.pl/web/koronawirus/wykaz-zarazenkoronawirusem-sars-cov-2. Accessed December 17, 2020.

3. Yang J, Zheng Y, Gou X, et al. Prevalence of comorbidities and its effects in patients infected with SARS-CoV-2: A systematic review and metaanalysis. Int J Infect Dis. 2020;94:91-95. doi:10.1016/j.ijid.2020.03.017
4. Epidemiology Working Group for NCIP Epidemic Response, Chinese Center for Disease Control and Prevention. The epidemiological characteristics of an outbreak of 2019 novel coronavirus diseases (COVID-19) in China [in Chinese]. Zhonghua Liu Xing Bing Xue Za Zhi. 2020;41(2):145-151. doi:10.3760/cma.j.issn.0254-6450.2020.02.003

5. Wang D, Hu B, Hu C, et al. Clinical characteristics of 138 hospitalized patients with 2019 novel coronavirus-infected pneumonia in Wuhan, China. JAMA. 2020;323(11):1061-1069. doi:10.1001/jama.2020.1585

6. Palaiodimos L, Kokkinidis DG, Li W, et al. Severe obesity, increasing age and male sex are independently associated with worse inhospital outcomes, and higher in-hospital mortality, in a cohort of patients with COVID-19 in the Bronx, New York. Metabolism. 2020;108:154262. doi:10.1016/j.metabol.2020.154262

7. Kim IC, Kim JY, Kim HA, Han S. COVID-19-related myocarditis in a 21-year-old female patient. Eur Heart J. 2020;41(19):1859. doi:10.1093/eurheartj/ehaa288

8. Huang C, Wang Y, Li X, et al. Clinical features of patients infected with 2019 novel coronavirus in Wuhan, China. Lancet. 2020;395(10223):497-506. doi:10.1016/S0140-6736(20)30183-5

9. Rojulpote C, Gonuguntla K, Patil S, Bhattaru A, Bravo PE. COVID-19 and the heart. Colomb Med (Cali). 2020;51(2):e4320. doi: $10.25100 / \mathrm{cm} . v 51 \mathrm{i} 2.4320$

10. Chen N, Zhou M, Dong X, et al. Epidemiological and clinical characteristics of 99 cases of 2019 novel coronavirus pneumonia in Wuhan, China: A descriptive study. Lancet. 2020;395(10223):507-513. doi:10.1016/S0140-6736(20)30211-7

11. Mauriello A, Sangiorgi G, Fratoni S, et al. Diffuse and active inflammation occurs in both vulnerable and stable plaques of the entire coronary tree: A histopathologic study of patients dying of acute myocardial infarction. J Am Coll Cardiol. 2005;45(10):1585-1593. doi:10.1016/j.jacc.2005.01.054

12. Madjid M, Vela D, Khalili-Tabrizi H, Casscells SW, Litovsky S. Systemic infections cause exaggerated local inflammation in atherosclerotic coronary arteries. Clues to the triggering effect of acute infections on acute coronary syndromes. Tex Heart Inst J. 2007;34(1):11-18.

13. Ye $Q$, Wang $B$, Mao J. The pathogenesis and treatment of the 'Cytokine Storm' in COVID-19. J Infect. 2020;80(6):607-613. doi:10.1016/j.jinf.2020.03.037

14. Xu Z, Shi L, Wang Y, et al. Pathological findings of COVID-19 associated with acute respiratory distress syndrome. Lancet Respir Med. 2020;8(4):420-422. doi:10.1016/S2213-2600(20)30076-X

15. Siripanthong B, Nazarian S, Muser D, et al. Recognizing COVID-19-related myocarditis: The possible pathophysiology and proposed guideline for diagnosis and management. Heart Rhythm. 2020;17(9):1463-1471. doi:10.1016/j.hrthm.2020.05.001 
16. Ho JS, Sia CH, Chan MY, Lin W, Wong RC. Coronavirus-induced myocarditis: A meta-summary of cases. Heart Lung. 2020;49(6):681-685. doi:10.1016/j.hrtIng.2020.08.013

17. Khatri A, Wallach F. Coronavirus disease 2019 (Covid-19) presenting as purulent fulminant myopericarditis and cardiac tamponade: A case report and literature review. Heart Lung. 2020;49(6):858-863. doi:10.1016/j.hrtIng.2020.06.003

18. Hua A, O'Gallagher K, Sado D, Byrne J. Life-threatening cardiac tamponade complicating myo-pericarditis in COVID-19. Eur Heart J. 2020;41(22):2130. doi:10.1093/eurheartj/ehaa253

19. Wrapp D, Wang N, Corbett KS, et al. Cryo-EM structure of the 2019-nCoV spike in the prefusion conformation. Science. 2020;367(6483):1260-1263. doi:10.1126/science.abb2507

20. Zou X, Chen K, Zou J, Han P, Hao J, Han Z. Single-cell RNA-seq data analysis on the receptor ACE2 expression reveals the potential risk of different human organs vulnerable to 2019-nCoV infection. Front Med. 2020;14(2):185-1932. doi:10.1007/s11684-020-0754-0

21. Zhang $P, Z$ hu L, Cai J, et al. Association of inpatient use of angiotensin-converting enzyme inhibitors and angiotensin II receptor blockers with mortality among patients with hypertension hospitalized with COVID-19. Circ Res. 2020;126(12):1671-1681. doi:10.1161/CIRCRESAHA.120.317134

22. Pascarella G, Strumia A, Piliego C, et al. COVID-19 diagnosis and management: A comprehensive review. J Intern Med. 2020;288(2):192-206. doi:10.1111/joim.13091

23. Yang $X, Y u Y, X u$ J, et al. Clinical course and outcomes of critically ill patients with SARS-CoV-2 pneumonia in Wuhan, China: A singlecentered, retrospective, observational study. Lancet Respir Med. 2020;8(5):475-481. doi:10.1016/S2213-2600(20)30079-5

24. Wu Z, McGoogan JM. Characteristics of and important lessons from the coronavirus disease 2019 (COVID-19) outbreak in China: Summary of a report of 72314 cases from the Chinese Center for Disease Control and Prevention. JAMA. 2020;323(13):1239-1242. doi:10.1001/jama.2020.2648

25. Guan W, Ni Z, Hu Y, et al. Clinical characteristics of coronavirus disease 2019 in China. N Engl J Med. 2020;382:1708-1720. doi:10.1056/NEJMoa2002032

26. Zhou Z, Zhao N, Shu Y, Han S, Chen B, Shu X. Effect of gastrointestinal symptoms in patients with COVID-19. Gastroenterology. 2020;158(8):2294-2297. doi:10.1053/j.gastro.2020.03.020

27. Ruan Q, Yang K, Wang W, Jiang L, Song J. Clinical predictors of mortality due to COVID-19 based on an analysis of data of 150 patients from Wuhan, China. Intensive Care Med. 2020;46(5):846-848. doi:10.1007/s00134-020-05991-x

28. Zhou $F, Y u T$, Du R, et al. Clinical course and risk factors for mortality of adult inpatients with COVID-19 in Wuhan, China: A retrospective cohort study. Lancet. 2020;395(10229):1054-1062. doi:10.1016/S0140-6736(20)30566-3

29. Driggin E, Madhavan MV, Bikdeli B, et al. Cardiovascular considerations for patients, health care workers, and health systems during the COVID-19 pandemic. J Am Coll Cardiol. 2020;75(18):2352-2371. doi:10.1016/j.jacc.2020.03.031

30. Li B, Yang J, Zhao F, et al. Prevalence and impact of cardiovascular metabolic diseases on COVID-19 in China. Clin Res Cardiol. 2020;109(5):531-538. doi:10.1007/s00392-020-01626-9

31. Kuba K, Imai Y, Ohto-Nakanishi T, Penninger JM. Trilogy of ACE2: A peptidase in the renin-angiotensin system, a SARS receptor, and a partner for amino acid transporters. Pharmacol Ther. 2010;128(1):119-128. doi:10.1016/j.pharmthera.2010.06.003

32. South AM, Diz DI, Chappell MC. COVID-19, ACE2 and the cardiovascular consequences. Am J Physiol Heart Circ Physiol. 2020;318(5):H1084-H1090. doi:10.1152/ajpheart.00217.2020

33. Dhakal BP, Sweitzer NK, Indik JH, Acharya D, William P. SARS-CoV-2 infection and cardiovascular disease: COVID-19 heart. Heart Lung Circ. 2020;29(7):973-987. doi:10.1016/j.hlc.2020.05.101

34. Vallamkondu J, John A, Wani WY, et al. SARS-CoV-2 pathophysiology and assessment of coronaviruses in CNS diseases with a focus on therapeutic targets. Biochim Biophys Acta Mol Basis Dis. 2020;1866(10):165889. doi:10.1016/j.bbadis.2020.165889

35. Varagic J, Ahmad S, Nagata S, Ferrario CM. ACE2: Angiotensin II/ angiotensin-(1-7) balance in cardiac and renal injury. Curr Hypertens Rep. 2014;16(3):420. doi:10.1007/s11906-014-0420-5
36. Banu N, Panikar SS, Leal LR, Leal AR. Protective role of ACE2 and its downregulation in SARS-CoV-2 infection leading to Macrophage Activation Syndrome: Therapeutic implications. Life Sci. 2020;256:117905. doi:10.1016/j.Ifs.2020.117905

37. Vaduganathan M, Vardeny O, Michel T, McMurray JJV, Pfeffer MA, Solomon SD. Renin-angiotensin-aldosterone system inhibitors in patients with Covid-19. N Engl J Med. 2020;382(17):1653-1659. doi:10.1056/NEJMsr2005760

38. Lu R, Zhao X, Li J, et al. Genomic characterisation and epidemiology of 2019 novel coronavirus: Implications for virus origins and receptor binding. Lancet. 2020;395(10224):565-574. doi:10.1016/S0140-6736(20)30251-8

39. Shi $Y, Y i$ Y, Li P, et al. Diagnosis of severe acute respiratory syndrome (SARS) by detection of SARS coronavirus nucleocapsid antibodies in an antigen-capturing enzyme-linked immunosorbent assay. J Clin Microbiol. 2003;41(12):5781-5782. doi:10.1128/jcm.41.12.5781-5782.2003

40. Kuster GM, Pfister O, Burkard T, et al. SARS-CoV2: Should inhibitors of the renin-angiotensin system be withdrawn in patients with COVID-19? Eur Heart J. 2020;41(19):1801-1803. doi:10.1093/eurheartj/ehaa235

41. Meng J, Xiao G, Zhang J, et al. Renin-angiotensin system inhibitors improve the clinical outcomes of COVID-19 patients with hypertension. Emerg Microbes Infect. 2020;9(1):757-760. doi:10.1080/2222175 1.2020.1746200

42. Liu G, Ma C, Yang $H$, Zhang PY. Transforming growth factor $\beta$ and its role in heart disease. Exp Ther Med. 2017;13(5):2123-2128. doi:10.3892/etm.2017.4246

43. Mahmudpour M, Roozbeh J, Keshavarz M, Farrokhi S, Nabipour I. COVID-19 cytokine storm: The anger of inflammation. Cytokine. 2020;133:155151. doi:10.1016/j.cyto.2020.155151

44. Chen $\mathrm{T}$, Wu D, Chen $\mathrm{H}$, et al. Clinical characteristics of 113 deceased patients with coronavirus disease 2019: Retrospective study. BMJ. 2020;368:m1091. doi:10.1136/bmj.m1091

45. Liu PP, Blet A, Smyth D, Li H. The science underlying COVID-19: Implications for the cardiovascular system. Circulation. 2020;142(1):68-78. doi:10.1161/CIRCULATIONAHA.120.047549

46. Amraei R, Rahimi N. COVID-19, renin-angiotensin system and endothelial dysfunction. Cells. 2020;9(7):1652. doi:10.3390/cells9071652

47. Bhatraju PK, Ghassemieh BJ, Nichols M, et al. Covid-19 in critically ill patients in the Seattle region - case series. $N$ Engl J Med. 2020;382(21):2012-2022. doi:10.1056/NEJMoa2004500

48. Zheng YY, Ma YT, Zhang JY, Xie X. COVID-19 and the cardiovascular system. Nat Rev Cardiol. 2020;17(5):259-260. doi:10.1038/s41569-020-0360-5

49. Goyal P, Choi JJ, Pinheiro LC, et al. Clinical characteristics of Covid-19 in New York City. N Engl J Med. 2020;382(24):2372-2374. doi:10.1056/ NEJMc2010419

50. Tsigaris P, Teixeira da Silva JA. Smoking prevalence and COVID-19 in Europe. Nicotine Tob Res. 2020;22(9):1646-1649. doi:10.1093/ntr/ntaa121

51. Lippi G, Lavie CJ, Sanchis-Gomar F. Cardiac troponin I in patients with coronavirus disease 2019 (COVID-19): Evidence from a meta-analysis. Prog Cardiovasc Dis. 2020;63(3):390-391. doi:10.1016/j.pcad.2020.03.001

52. Shastri MD, Shukla SD, Chong WC, et al. Smoking and COVID-19: What we know so far. Respir Med. 2021;176:106237. doi:10.1016/j. rmed.2020.106237

53. Bangalore S, Sharma A, Slotwiner A, et al. ST-segment elevation in patients with Covid-19 - a case series. $N$ Engl J Med. 2020;382(25):2478-2480. doi:10.1056/NEJMc2009020

54. Meyer P, Degrauwe S, Van Delden C, Ghadri JR, Templin C. Typical takotsubo syndrome triggered by SARS-CoV-2 infection. Eur Heart J. 2020;41(19):1860. doi:10.1093/eurheartj/ehaa306

55. De Filippo O, D’Ascenzo F, Angelini F, et al. Reduced rate of hospital admissions for ACS during Covid-19 outbreak in Northern Italy. N Engl J Med. 2020;383(1):88-89. doi:10.1056/NEJMc2009166

56. Roffi M, Guagliumi G, Ibanez B. The obstacle course of reperfusion for ST-segment-elevation myocardial infarction in the COVID-19 pandemic. Circulation. 2020;141(24):1951-1953. doi:10.1161/CIRCULATIONAHA.120.047523

57. Daoulah A, Hersi AS, Al-Faifi SM, et al. STEMI and COVID-19 pandemic in Saudi Arabia. Curr Probl Cardiol. 2021;46(3):100656. doi:10.1016/j.cpcardiol.2020.100656 
58. Sala S, Peretto G, Gramegna M, et al. Acute myocarditis presenting as a reverse Tako-Tsubo syndrome in a patient with SARSCoV-2 respiratory infection. Eur Heart J. 2020;41(19):1861-1862. doi:10.1093/eurheartj/ehaa286

59. Yao XH, Li TY, He ZC, et al. A pathological report of three COVID-19 cases by minimal invasive autopsies [in Chinese]. Zhonghua Bing Li Xue Za Zhi. 2020;49(5):411-417. doi:10.3760/cma.j.cn112151-20200312-00193

60. Tavazzi G, Pellegrini C, Maurelli M, et al. Myocardial localization of coronavirus in COVID-19 cardiogenic shock. Eur J Heart Fail. 2020;22(5):911-915. doi:10.1002/ejhf.1828

61. Fox SE, Akmatbekov A, Harbert JL, Li G, Brown Q, Vander Heide RS Pulmonary and cardiac pathology in Covid-19: The first autopsy series from New Orleans. medRxiv preprint. 2020. doi:10.1101/2020.04.06.20050575

62. Kim IC, Kim JY, Kim HA, Han S. COVID-19-related myocarditis in a 21-year-old female patient. Eur Heart J. 2020;41(19):1859. doi:10.1093/eurheartj/ehaa288

63. Gawałko M, Kapłon-Cieślicka A, Hohl M, Dobrev D, Linz D. COVID-19 associated atrial fibrillation: Incidence, putative mechanisms and potential clinical implications. Int J Cardiol Heart Vasc. 2020;30:100631. doi:10.1016/j.ijcha.2020.100631

64. Si D, Du B, Ni L, et al. Death, discharge and arrhythmias among patients with COVID-19 and cardiac injury. CMAJ. 2020;192(28):E791-E798. doi:10.1503/cmaj.200879

65. Grein J, Ohmagari N, Shin D, et al. Compassionate use of remdesivir for patients with severe Covid-19. N Engl J Med. 2020;382(24):2327-2336. doi:10.1056/NEJMoa2007016

66. Azarkish M, Far VL, Eslami M, Mollazadeh R. Transient complete heart block in a patient with critical COVID-19. Eur Heart J. 2020;41(22):2131. doi:10.1093/eurheartj/ehaa307

67. Ng KHL, Wu AKL, Cheng VCC, et al. Pulmonary artery thrombosis in a patient with severe acute respiratory syndrome. Postgrad Med J. 2005;81(956):e3. doi:10.1136/pgmj.2004.030049

68. Léonard-Lorant I, Delabranche $X$, Séverac $F$, et al. Acute pulmonary embolism in patients with COVID-19 at CT angiography and relationship to d-dimer levels. Radiology. 2020;296(3):E189-E191. doi:10.1148/radiol.2020201561

69. Wichmann D, Sperhake JP, Lütgehetmann M, et al. Autopsy findings and venous thromboembolism in patients with COVID-19: A prospective cohort study. Ann Intern Med. 2020;173(4):268-277. doi:10.7326/M20-2003

70. Ackermann $M$, Verleden SE, Kuehnel M, et al. Pulmonary vascular endothelialitis, thrombosis, and angiogenesis in Covid-19. $N$ Engl J Med. 2020;383(2):120-128. doi:10.1056/NEJMoa2015432

71. Dolhnikoff $M$, Duarte-Neto AN, de Almeida Monteiro RA, et al. Pathological evidence of pulmonary thrombotic phenomena in severe COVID-19. J Thromb Haemost. 2020;18(6):1517-1519. doi:10.1111/jth.14844

72. Klok FA, Kruip MJHA, van der Meer NJM, et al. Incidence of thrombotic complications in critically ill ICU patients with COVID-19. Thromb Res. 2020;191:145-147. doi:10.1016/j.thromres.2020.04.013

73. Iba T, Levy JH, Levi M, Thachil J. Coagulopathy in COVID-19. J Thromb Haemost. 2020;18(9):2103-2109. doi:10.1111/jth.14975

74. Zulfiqar $A A$, Lorenzo-Villalba $N$, Hassler $P$, Andrès $E$. Immune thrombocytopenic purpura in a patient with Covid-19. NEngl J Med. 2020:382(18):e43. doi:10.1056/NEJMc2010472

75. Yerasi C, Case BC, Forrestal BJ, et al. Treatment of ST-segment elevation myocardial infarction during COVID-19 pandemic. CardiovasC Revasc Med. 2020;21(8):1024-1029. doi:10.1016/j.carrev.2020.05.027

76. Sakabe M, Yoshioka R, Fujiki A. Sick sinus syndrome induced by interferon and ribavirin therapy in a patient with chronic hepatitis $C$. J Cardiol Cases. 2013;8(6):173-175. doi:10.1016/j.jccase.2013.08.002

77. Guzik TJ, Mohiddin SA, Dimarco A, et al. COVID-19 and the cardiovascular system: Implications for risk assessment, diagnosis, and treatment options. Cardiovasc Res. 2020;116(10):1666-1687. doi:10.1093/cvr/cvaa106 